\title{
Antarctic Sea Ice-a Habitat for Extremophiles
}

\section{N. Thomas ${ }^{1 *}$ and G. S. Dieckmann ${ }^{2}$}

The pack ice of Earth's polar oceans appears to be frozen white desert, devoid of life. However, beneath the snow lies a unique habitat for a group of bacteria and microscopic plants and animals that are encased in an ice matrix at low temperatures and light levels, with the only liquid being pockets of concentrated brines. Survival in these conditions requires a complex suite of physiological and metabolic adaptations, but sea-ice organisms thrive in the ice, and their prolific growth ensures they play a fundamental role in polar ecosystems. Apart from their ecological importance, the bacterial and algae species found in sea ice have become the focus for novel biotechnology, as well as being considered proxies for possible life forms on icecovered extraterrestrial bodies.

$\rho^{2}$ ea ice is an ephemeral feature of polar regions, but also of the Baltic, Caspian, and Okhotsk Seas. At its maximum, it covers $13 \%$ of the Earth's surface area (1), making it one of the major biomes on the planet, similar, in terms of area, to that of deserts and tundra (2). The largest expanse of sea ice occurs in the Southern Ocean where, during winter, 20 million square kilometers are blanketed by an ice cover approximately $1 \mathrm{~m}$ thick. Unlike freshwater ice, frozen seawater forms a semisolid matrix, permeated by a network of channels and pores. These vary in size from a few micrometers to millimeters, and are filled with brine formed from expelled salts as the ice crystals freeze together (3). It is within this labyrinth that the sea-ice organisms live (Fig. 1).

Sea ice is dominated by strong gradients in temperature, salinity, space, and light (Fig. 2 ). These properties and the morphology of the brine channel system are highly variable, being ultimately governed by air temperature and snow cover. Large seasonal and even diurnal differences in ice properties occur (3). Small-scale variations in ice morphology are compounded by rafting of ice floes and deformation, which impart a tremendous spatial heterogeneity to any sea-ice zone, even within a single floe.

Change in salinity is the dominant factor in external chemistry to influence the biological assemblages within the sea ice. Owing to the closed or semiclosed pore system within the ice, diffusion rates of dissolved gases and exchange of inorganic nutrients are greatly retarded (4). Hyperoxic brines depleted in carbon dioxide, high concentrations of dis-

15chool of Ocean Sciences, University of WalesBangor, Menai Bridge, Anglesey, UK, LL59 5EY. ${ }^{2}$ Alfred Wegener Institute for Polar and Marine Research, Am Handelshafen 12, D- 27570 Bremerhaven, Germany.

*To whom correspondence should be addressed. Email: d.thomas@bangor.ac.uk solved organic matter, low concentrations of nutrients, high ammonia concentrations, and elevated $\mathrm{pH}$ values are all a consequence of biological activity within the confined system. Physiological and biochemical adaptations of the sea-ice biota accommodate swift transitions between water and ice and the subsequent rapid (hours or days) physical and chemical changes.

\section{Biological Assemblages}

Many planktonic organisms, including viruses, bacteria, algae, protists, flatworms, and small crustaceans, stick to, or are caught between, ice crystals as they rise through the water when surface waters freeze in autumn. Subsequently, as the ice grows and consolidates, the organisms become trapped within the brine channels $(3,5)$. Hence, a diverse group of organisms (Fig. 3) is almost instantaneously confined to a new habitat that is quite different from the one from which they were recruited.

The most conspicuous organisms in the ice are pennate diatoms (unicellular photosynthetic microalgae), which reach such concentrations that their photosynthetic pigments discolor the ice brown (Fig. 1). Diatom standing stocks up to $1000 \mu \mathrm{g}$ of chlorophyll per liter have been measured in sea ice, which compare with typical values of 0 to $5 \mu \mathrm{g}$ of chlorophyll per liter for surface waters in the Southern Ocean. Primary production in Antarctic sea ice (63 to $70 \mathrm{Tg} \mathrm{C}$ per year) is estimated to contribute $5 \%$ to the total annual primary production (1300 $\mathrm{Tg} \mathrm{C}$ per year) in the sea ice-influenced zone of the Southern Ocean (2). This apparently minor contribution is in fact highly significant, in that the sea-ice organisms provide a concentrated food source that is asynchronous with, and that extends, the short period of primary production in the water column. Heterotrophic protists and metazoans, such as small copepods, can accumulate in sea ice at concen- trations ranging from hundreds to thousands of individuals per liter; several orders of magnitude higher than those in the water. The smaller protozoans and metazoans graze the dense growths of bacteria and algae, whereas the narrow brine channels exclude larger species from exploiting these food sources (6). The distribution and feeding activity of krill, the pivotal organism in the Southern Ocean food web, is strongly correlated with pack ice cover (7). Krill stocks can exceed 1.5 billion tons (c.f., the total mass of people approximates 0.5 billion tons) and are the primary food for squid, penguins, seals, and baleen whales. Krill feed on phytoplankton during the summer; in winter, when the water is virtually devoid of plankton, sea-ice algae are the primary source of food for krill overwintering in ice-covered regions.

Bacteria are conspicuous in sea-ice assemblages (Fig. 3), entering the ice mostly on the surfaces of larger organisms (5). As temperatures drop, there is a gradual transition in bacterial populations from the diverse mixed species inoculums originating in seawater to psychrophilic species (minimum growth temperature $\leq 0^{\circ} \mathrm{C}$, optimal growth temperature $<15^{\circ} \mathrm{C}$, and maximum growth temperature $<20^{\circ} \mathrm{C}$ ) (8). The growth of psychrophilic heterotrophs is fueled by large pools of dissolved organic matter consisting largely of carbohydrates produced by the death and lysis of sea-ice organisms, as well as by the exudation of organic polymers by algae and bacteria $(9,10)$.

\section{Low Temperature}

The capacity to survive at temperatures down to $-20^{\circ} \mathrm{C}$ is a prerequisite for life in sea ice. Many marine microorganisms, particularly algae and protozoans, form robust stress resistant cysts at specific stages of their life histories, probably in response to falling temperatures as ice forms. Limited experimental work done to date indicates that, although this may be a strategy for some species, other factors, such as limitations in inorganic nutrient availability, may be more important signals for cyst formation (11).

The most critical metabolic requirement at low temperatures is maintenance of functional lipid membranes. The fatty acid composition of membrane phospholipids regulates the degree of membrane fluidity; hence, an increase in the proportion of unsaturated fatty acids, a decrease in average chain length and an increase in polyunsaturated fatty acids (PUFAs) is central to retaining membrane 


\section{SCIENCE'S COMPASS}

fluidity at low temperatures $(8,12)$. A novel enzyme family, the polyketide synthases, which are active at low temperatures and are required for the biosynthesis of PUFAs, have recently been found in several psychrophilic bacteria isolated from sea ice $(13,14)$. PUFAs are not only essential to the organisms themselves, but are also an important component in the diets of grazing organisms and of fish and mammals. In winter, PUFAs production is probably enhanced, so that a very different quality of food is released into the food web than in other seasons when PUFA synthesis is lower.

Most studies on lipid and membrane metabolism have been conducted on psychrophilic bacteria, although regulation of membrane lipid composition in sea-ice diatoms has been shown to be essential for efficient electron transport under low temperatures, low irradiances, and nitrogen limitation (15). Low temperatures are generally coupled with low irradiance in sea ice. Light also regulates the fluidity of the thylakoid membrane and is indirectly responsible for cold acclimation of photosystem II in sea-ice diatoms. At low irradiances the proportion of PUFAs in monogalactosyldiacylglycerol, the main lipid class of thylakoid membranes, increases. PUFAs support the interactions between primary (QA) and secondary (QB) electron acceptors and thus the velocity of electron flow. Apart from the influence of lipids on electron transport, they also regulate the structure of thylakoid membranes. During periods of nitrogen limitation, protein biosynthesis is affected, and the loss of bilayer stabilizing proteins and pigments could disrupt chloroplast membranes and, consequently, photosynthe- sis. But in psychrophilic diatoms, membrane protein loss caused by nutrient limitation can be compensated for by the production of bilayer forming fatty acids, such as digalactosyldiaglycerol and phosphatidylglycerol (15).

Psychrophilic enzymes are characterized by a high catalytic efficiency at low temperatures, high degrees of thermolability, and increased structural flexibility for better substrate access. At low temperatures, protein folding has a critical role in conferring cold adaptability $(8,16)$. Again, most of the work has been conducted on psychrophilic bacteria from sea ice, which have been found to have highly cold-adapted and salt-tolerant proteases, $\beta$-galactosidases, phosphatase, and amylase (17). An alternative mechanism for cold adaptation is required for one of the key enzymes in photosynthesis and the most abundant enzyme in plants, ribulose-1,5-bisphosphate carboxylase/oxygenase (RUBISCO). RUBISCO has poor catalytic efficiency at low temperatures in isolated psychrophilic microalgae. Nevertheless, its activity is maintained, as temperatures drop, by an increase in the concentrations of the enzyme (16).

The growth of sea-ice microorganisms at low temperatures can also be limited by reduced affinity for substrates, even though these may be available at much higher concentrations in the ice matrix than in seawater (17). However, this does not hold true for all substrates, and, for example, the affinity of microalgae and bacteria for ammonium shows no temperature dependence, whereas in the same organisms, nitrate affinity falls as the temperature becomes suboptimal for growth (18). Consequently, there may be an increased dependence on ammonium as an inorganic nitrogen source for algae and bacteria at low temperatures. This dependency may be enhanced by the elevated $\mathrm{pH}$ in sea-ice brines increasing the dissociation of ammonium to ammonia that can diffuse directly into cells. $(18,19)$. Ammonium levels can be as high as 150 $\mu \mathrm{M}$ in sea ice (in seawater the values are generally $<1 \mu \mathrm{M}$ ), and such concentrations will also inhibit the assimilation of nitrate, owing to the inactivation of nitrate reductase by glutamine produced during the assimilation of ammonia (20).

\section{Salinity}

Freezing tolerance is often accompanied by tolerance to dehydration caused by a lack of free water at low temperatures or high salinities. Mechanisms of freezing and dehydration tolerance in plants may involve common gene products and regulatory pathways (21), and it is likely that these occur in sea-ice organisms too. Dehydration, caused by the high brine salinities, is a major stressor for ice-trapped organisms, which may experience salinities three times that of seawater (Fig. 4). Conversely, when the ice melts, the released organisms will be suddenly exposed to hyposaline conditions close to freshwater values. Under salinity stress, the lipid packing and the proportions of fatty acids (monounsaturated and branched-chain fatty acids) of psychrophilic bacteria at low temperatures are regulated (22), and salt-tolerant enzymes that function over a wide range of salinities have also been characterized from psychrophilic isolates $(17,22)$.

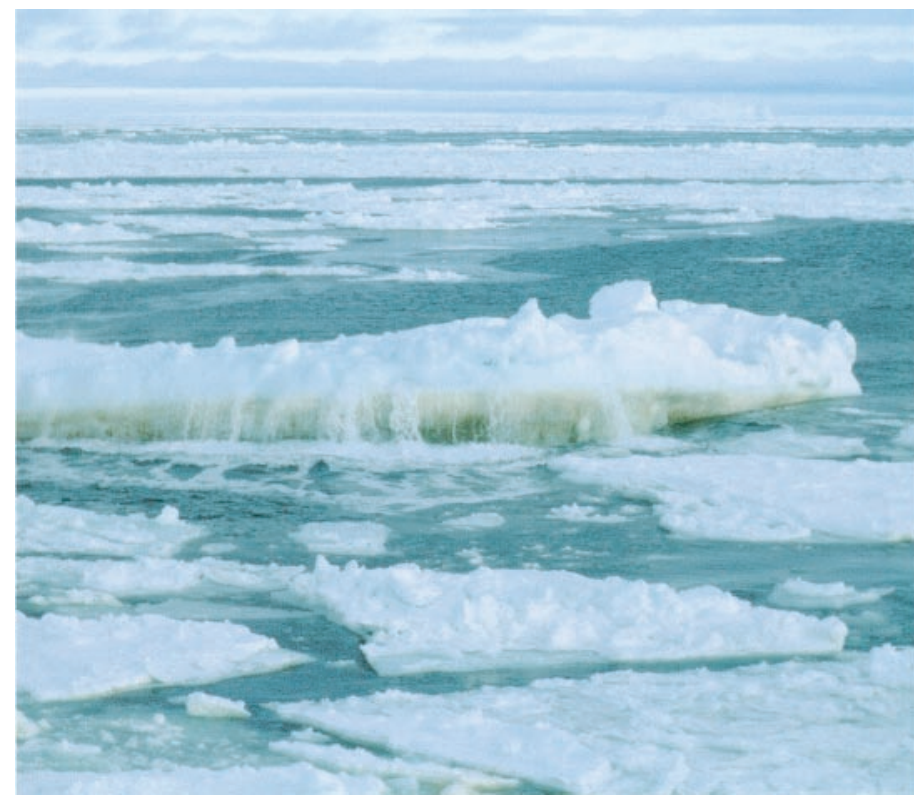

Fig. 1. Sea-ice diatoms can reach such densities that their photosynthetic pigments color the underside of ice floes brown. The sea-ice organisms grow throughout the ice in brine channels, the brown

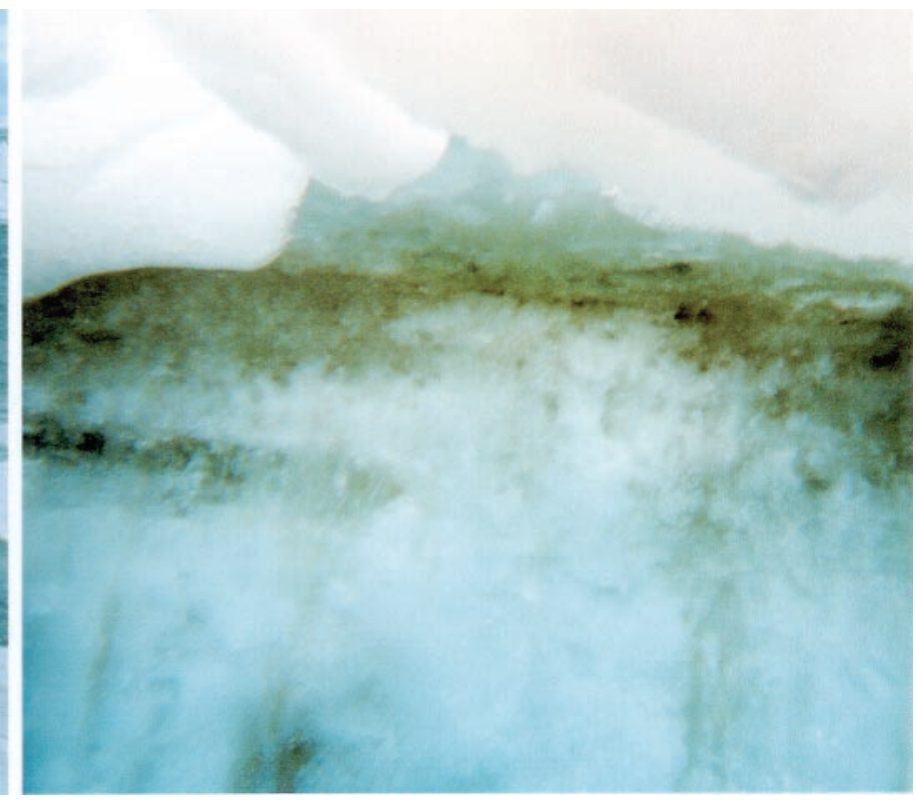

vertical lines in the image on the right, as well as in porous ice layers, which can be seen as dark brown horizontal layers. [Photographs by D. N. Thomas] 


\section{SCIENCE'S COMPASS}

Surviving osmotic stress requires the adjustment of cellular concentrations of osmolytes, so as to restore the osmotic balance between the external medium and the inside of the cells. In response to the changing external osmotic pressure osmolytes, including inorganic ions and organic solutes (such as proline, mannitol, and glycine betaine), are accumulated or synthesized in hypersaline conditions and broken down or released during hyposaline shock. An interesting osmolyte and cryoprotectant found at high concentrations in sea-ice algae is dimethylsulfonioproprionate (DMSP) the precursor of volatile dimethyl sulfide system damage (23). What happens to respiration at low irradiance and low temperature is not well understood, although it has been suggested to be a key regulatory factor in acclimation to low light levels (23).

Although the evidence is inconclusive, during periods of very low light, especially in winter, sea-ice algae may be able to switch from autotrophy to heterotrophic uptake of organic matter (5). Recently, it has been shown that a single gene encoding a glucose transporter can be introduced into photosynthetic marine diatoms to induce a fundamental change in metabolism that allows the organisms to grow on glucose in the absence of light (26). Similar metabolic shifts may occur in naturally occurring sea-ice diatoms during changing environmental conditions.

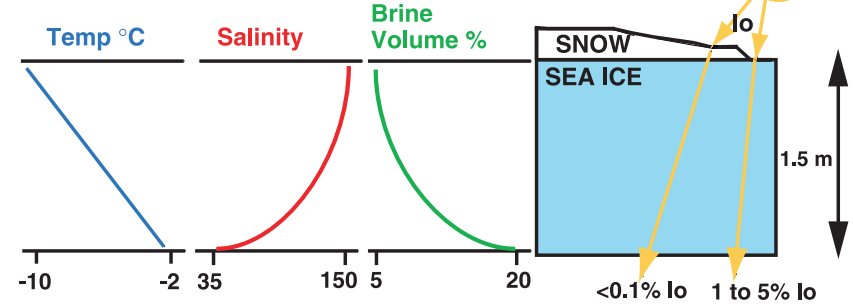

Fig. 2. Gradients of temperature, salinity, and brine volume are established across an ice floe. The underside is always at the freezing point of seawater $-1.8^{\circ} \mathrm{C}$ and the top of the ice close to air temperature, although this is largely dependent on snow cover. The illustration shows how snow cover can significantly reduce the amount of incident irradiance (lo). [Adapted from (3), with permission from Springer-Verlag]

(DMS) (23). DMS is released on the cleavage of DMSP and in the atmosphere is oxidized to $\mathrm{SO}_{2}$, methane sulfonate, and non-sea salt sulfate, which act as cloud condensation nuclei. High accumulations of biogenically derived DMS from the ice can therefore play an important role in cloud formation and, hence, climate regulation.

\section{Low-Light Conditions}

In general, ice algae have photosynthetic mechanisms that are saturated at low incident irradiances and have high photosynthetic efficiencies. Sea ice, especially when covered with snow, is an effective barrier to light transmission, and sea-ice algae have to be physiologically geared to living at low light. For example, oxygen-evolving marine photolithotrophs are able to grow in very low light, down to $10 \mathrm{nmol}$ photon per square meter per second (24). Such photoacclimation requires the quantitative regulation of photosynthetic pigments and changes in pigment composition. Concentrations of the photosynthetic accessory pigment fucoxanthin increase, as it is highly effective at absorbing wavelengths that can penetrate sea ice (25). Low lightacclimated sea-ice algae often display enhanced levels of photoinhibition, a reduction of photosynthetic efficiency at high irradiances, which can be associated with photo- protective, mycosporine-like amino acid, porphyra-334, have been measured in sea-ice diatom samples. UV radiation-induced damage to RNA transcription and DNA replication has been seen in ice diatoms, as has UV-B radiation damage to photosystem II in ice algae, accounting for most of the observed UV-B-specific inhibition of carbon assimilation by whole cells (28). The consequences of UV-B-induced effects are unlikely to be manifested by reduced productivity of the ice communities because of the protection afforded by the ice matrix, but more subtle changes in species composition and or succession patterns of species are possible (27).

\section{Dissolved Gases}

Physiological differences in inorganic carbon uptake may be a decisive factor controlling species diversity in sea-ice algal blooms. A consequence of high photosynthetic activity in ice is a depletion of inorganic carbon almost to exhaustion and an increase in $\mathrm{pH}$ in the brines to $\mathrm{pH} 11$ (normal seawater has a $\mathrm{pH}$ of 8) (4). Low inorganic carbon concentrations also alter the stable carbon isotope signatures of sea-ice diatoms to such a degree that material sedimenting from the ice has quite different carbon stable isotope values from those of cells that have grown in surface waters (4). This has important consequences for the use of stable isotopes in marine sediments for the reconstruction of past seawater temperatures or ice cover.

Oxygen stoichiometry in brine is also largely unknown, and oxygen measurements in sea-ice brine reservoirs are rare. Those made to date indicate that hyperoxia

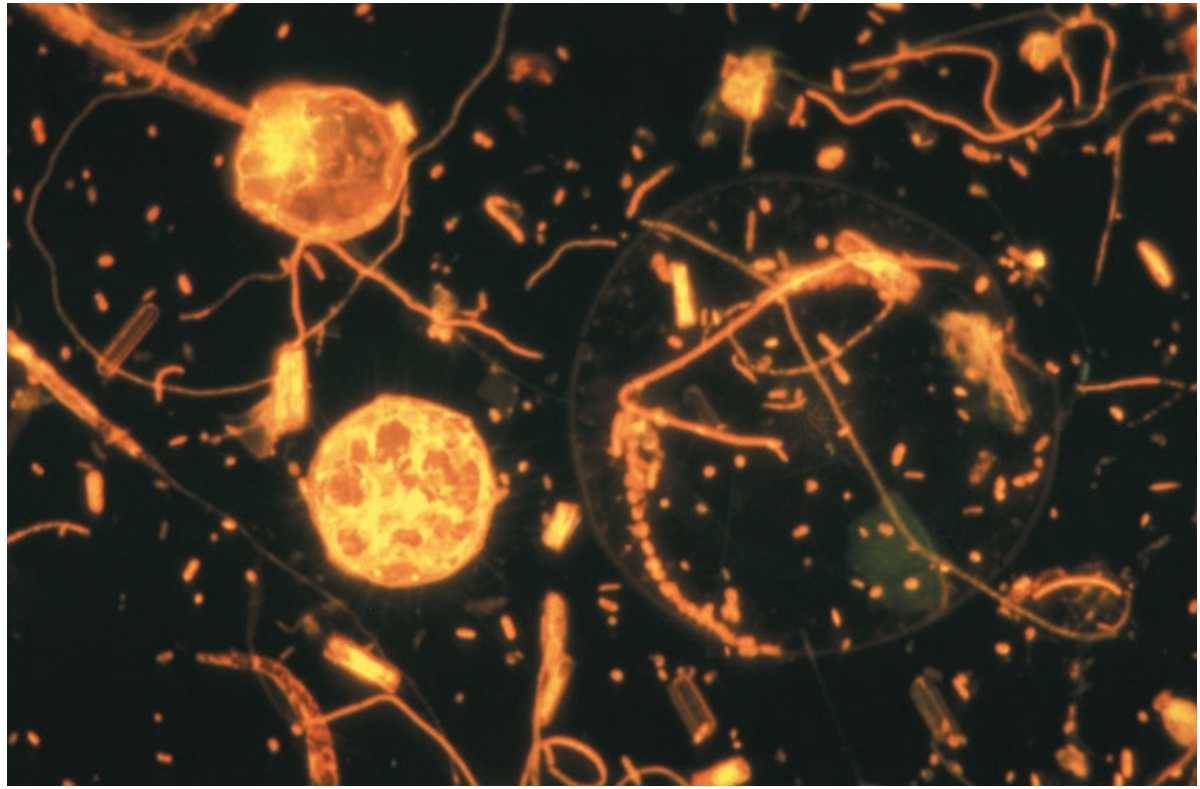

Fig. 3. Psychrophilic bacteria. The small fluorescing dots and rods in the photomicrograph grow to high numbers in sea ice. Their productivity, in terms of carbon biomass, generally lags behind the primary production of sea-ice algae (large fluorescing circles), except in winter, when bacterial activity may exceed that of the primary producers. [Photograph by S. Grossmann] 


\section{SCIENCE'S COMPASS}

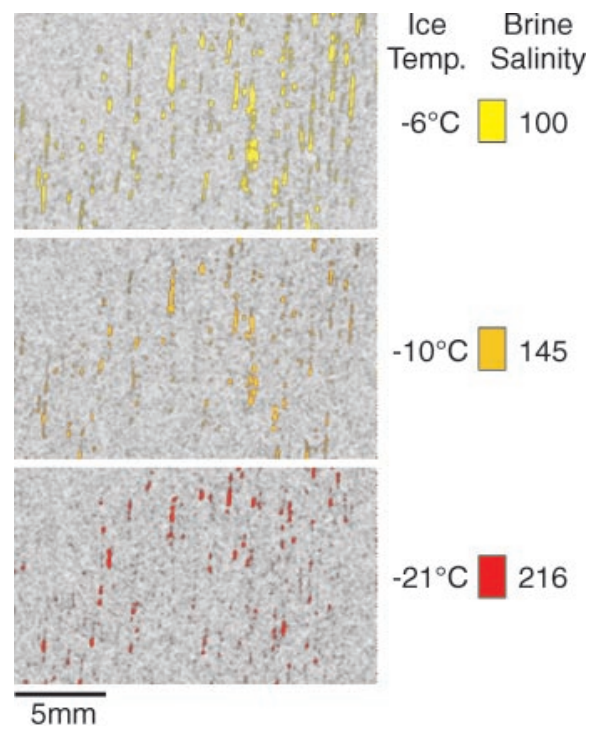

Fig. 4. Seawater has a salinity (in kilograms of salts per kilogram water) of 34 . As ice consolidates, salts from the seawater are expelled to form brines that collect in brine channels and pores (40). This series of color-enhanced magnetic resonance images of the same piece of sea ice shows how the pore space and size reduces with decreasing temperature, with a corresponding increase in salinity of brines contained within the pores. [Adapted from (41), with permission from Elsevier Science]

is coupled with low inorganic carbon concentrations (4). Hyperoxia is rare in marine systems and is potentially toxic, owing to the production of reactive oxygen species and hydroxyl radicals (29). Toxic photochemical products are also produced by the interaction of UV-B radiation, oxygen, and certain organic molecules. Little is known about how sea-ice organisms cope with oxidative stress, but isolated sea-ice diatoms have highly active antioxidative enzymes, such as catalase, glutathione peroxidase, and glutathione reductase. These protective enzymes function over the range of irradiance and temperatures where the greatest photosynthetic and respiratory activity in the ice takes place (30).

\section{Creation of Microenvironments in the Ice}

In addition to intercellular responses to the low temperatures, many sea-ice diatoms release ice-active substances, thought to be glycoproteins, although they still need to be fully characterized (31). Glycoproteins may modify the surfaces and optical properties of ice around the diatoms and/or increase the ability of cells to adhere to ice crystals (31). Sea-ice algae and bacteria are known to produce large amounts of soluble organic matter, much of which is in the form of extracellular polymeric substances (EPS) $(9,10)$, consisting mainly of polysaccharides. In dense accumulations of algae and bacteria, it is possible that matrices akin to biofilms develop within the brine channels. The viscous biofilm surrounding the cells must significantly alter the immediate physicochemical environment. Although almost nothing is known about biofilms in sea ice, it can reasonably be speculated that they protect organisms within ice floes from ice-crystal damage, buffer against $\mathrm{pH}$ and salinity changes, and ameliorate other chemical stresses. EPS have been shown to significantly alter the morphology of brine pore space and, hence, the permeability for solutes and microorganisms within the ice matrix (10). EPS probably also provide structures on which organisms grow and move, and may offer protection from grazers.

\section{From Sea Ice to Space}

In recent years, the study of sea-ice organisms has intensified, fueled in part by the realization that the physiological and biochemical acclimations that microorganisms thriving in the ice have to undergo may have considerable potential for biotechnological applications. Current preoccupations include the production of PUFAs for aquaculture, livestock, and human food, and the use of cold-adapted enzymes for a host of industries, ranging from cleaning agents to food processing $(12,23)$. Nevertheless, of most pressing concern is how climate change will affect seasonal changes in the extent of sea ice. Any change in sea-ice extent and, therefore, in the distribution of sea-ice organisms will have significant consequences to seasonal grazing behavior and life history strategies of key organisms, such as krill, and thus for the entire Antarctic food web. But some of the keenest interest in sea-ice organism biology comes from the new breed of astrobiologists who are enthusiastically scrutinizing the ice-covered seas of Jupiter's moons Europa (33) and Ganymede (34), as well as Mars (35), and speculating about the sea ice-covered neoproterozoic "snowball earth" (36, 37). Despite the tantalizing lure to compare the brown coloration observed on Europa's surface to diatom-colored sea ice (37), the extraterrestrial ice systems, $10 \mathrm{~s}$ to $100 \mathrm{~s} \mathrm{~km}$ thick, are substantially different from the 1to 10-m-thick ice we know from Earth's polar oceans. If life forms do exist, or have existed on these bodies, it seems that they will be quite unlike those that dominate the sea ice found on Earth today $(38,39)$.

\section{References and Notes}

1. C. L. Parkinson, P. Gloersen, in Atlas of Satellite $\mathrm{Ob}$ servations Related to Global Change, R. J. Gurney, J. -L. Foster, and C. L. Parkinson, Eds. (Cambridge Univ. Press, Cambridge, 1993).

2. M. P. Lizotte, Am. Zool. 41, 57 (2001).

3. H. Eicken, Polar Biol. 12, 3 (1992).

4. M. Gleitz et al., Mar. Chem. 51, 81 (1995).

5. A. C. Palmisano, D. L. Garrison, in Antarctic Microbiology, E. I. Friedman, Ed. (Wiley-Liss, New York, 1993), pp. 167-218.

6. C. Krembs, R. Gradinger, M. Spindler, J. Exp. Mar. Biol. Ecol. 243, 55 (2000).

7. V. Loeb et al., Nature 387, 897 (1997).

8. D. S. Nichols, P. D. Nichols, T. A. McMeekin, Science Progr. 78, 311 (1995).

9. D. N. Thomas et al., Ann. Glaciol. 33, 297 (2001).

10. C. Krembs, K. Junge, J. W. Deming, H. Eicken, ASLO 2001 Aquatic Sciences Meeting, Albuquerque, NM, 12 to 16 February 2001; available at www.aslo.org/ albuquerque2001/cs25.html.

11. D. K. Stoecker, D. E. Gustafson, M. M. D. Black, C. T. Baier, J. Phycol. 34, 60 (1998).

12. N. J. Russell, Comp. Biochem. Physiol. 118, 489 (1997).

13. J. P. Bowman et al., Int. J. Syst. Bacteriol. 48, 1171 (1998).

14. J. G. Metz et al., Science 293, 290 (2001).

15. T. Mock, B. M. A. Kroon, personal communication.

16. N. Devos, M. Ingouff, R. Loppes, R. F. Matagne, J. Phycol. 34, 655 (1998).

17. L. R. Pomeroy, W. J. Wiebe, Aquat. Microb. Ecol. 23, 187 (2000).

18. D. S. Reay, D. B. Nedwell, J.Priddle, J. C. Ellis-Evans, Appl. Environ. Microbiol. 65, 2577 (1999).

19. J. A. Raven, B. Wollenweber, L. L Handley, New Phytol. 121, 19 (1992).

20. J. C. Priscu, C. W. Sullivan, Antarct. Res. Ser. 73, 147 (1998).

21. M. F. Thomashow, Plant Physiol. 125, 89 (2001).

22. D. S. Nichols, J. Olley, H. Garda, R. R. Brenner, T. A. McMeekin, Appl. Environ. Microbiol. 66, 2422 (2000).

23. G. O. Kirst, C. Wiencke, J. Phycol. 31, 181 (1995).

24. J. A. Raven, J. E. Kubler, J. Beardall, J. Mar. Biol. Assoc. U.K. 80, 1 (2000).

25. M. P. Lizotte, D. H. Robinson, C. W. Sullivan, Antarct. Res. Ser. 73, 93 (1998).

26. L. A. Zaslavskaia et al., Science 292, 2073 (2001).

27. W. F. Vincent, S. Roy, Environ. Rev. 1, 1 (1993).

28. B. Prézelin, M. A. Moline, H. A. Matlick, Antarct. Res. Ser. 73, 45 (1998).

29. J. A. Raven, Palaeogeogr. Palaeoclimatol. Paleaeoecol. 97, 19 (1991).

30. R. Schrieck, Rep. Polar Res. 349, 1 (2000)

31. J. A. Raymond, Polar Biol. 23, 721 (2000).

32. D. Nichols et al., Curr. Opin. Biotechnol. 10, 240 (1999).

33. M. G. Kivelson et al., Science 289, 1340 (2000).

34. T. B. McCord, G. B. Hansen, C. A. Hibbitts, Science 292, 1523 (2001).

35. M. C. Malin, K. S. Edgett, Science 288, 2330 (2000).

36. P. F. Hoffman, A. J. Kaufman, G. P. Halverson, D. P. Schrag, Science 281, 1342 (1998).

37. R. B. Hoover, F. Hoyle, N. C. Wickramasinghe, M. J. Hoover, S. AlMufti, Earth Moon Planets 35, 19 (1986).

38. E. J. Gaidos, K. H. Nealson, J. L. Kirschvink, Science 284, 1631 (1999).

39. C. F. Chyba, K. P. Hand, Science 292, 2026 (2001).

40. G. F. N. Cox, W. F. Weeks, J. Glaciol. 32, 371 (1986).

41. H. Eicken, C. Bock, R. Wittig, H. Miller, H.-O. Poertner, Cold Reg. Sci. Technol. 31, 207 (2000).

42. This work was partly supported by NERC, the British Council, the Nuffield Foundation, and the Hanse Institute of Advanced Study. We thank T. Mock, H. Eicken, and C. Krembs for their advice. 